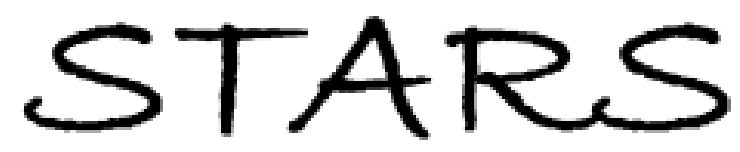

University of Central Florida

STARS

$1-1-2005$

\title{
Polarization-independent and fast-response phase modulation using a normal-mode polymer-stabilized cholesteric texture
}

\author{
Yi-Hsin Lin \\ University of Central Florida \\ Hongwen Ren \\ University of Central Florida \\ Yun-Hsing Fan \\ University of Central Florida \\ Yung-Hsun Wu \\ University of Central Florida \\ Shin-Tson Wu \\ University of Central Florida \\ Find similar works at: https://stars.library.ucf.edu/facultybib2000 \\ University of Central Florida Libraries http://library.ucf.edu
}

This Article is brought to you for free and open access by the Faculty Bibliography at STARS. It has been accepted for inclusion in Faculty Bibliography 2000s by an authorized administrator of STARS. For more information, please contactSTARS@ucf.edu.

\section{Recommended Citation}

Lin, Yi-Hsin; Ren, Hongwen; Fan, Yun-Hsing; Wu, Yung-Hsun; and Wu, Shin-Tson, "Polarization-independent and fast-response phase modulation using a normal-mode polymer-stabilized cholesteric texture" (2005). Faculty Bibliography 2000s. 5409.

https://stars.library.ucf.edu/facultybib2000/5409

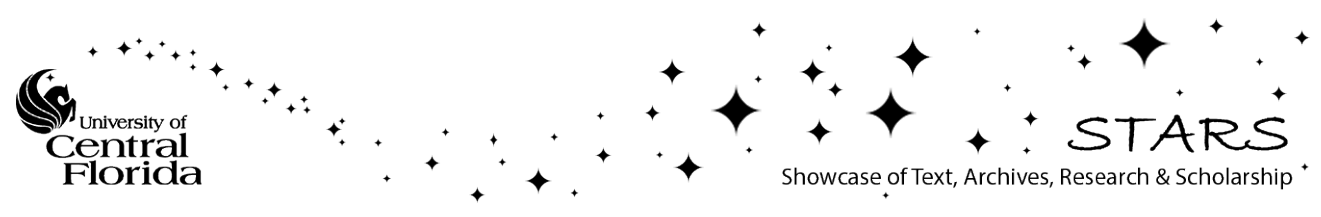




\section{Polarization-independent and fast-response phase modulation using a normal-mode polymer-stabilized cholesteric texture}

Cite as: J. Appl. Phys. 98, 043112 (2005); https://doi.org/10.1063/1.2037191

Submitted: 09 May 2005. Accepted: 25 July 2005. Published Online: 25 August 2005

Yi-Hsin Lin, Hongwen Ren, Yun-Hsing Fan, Yung-Hsun Wu, and Shin-Tson Wu

\section{ARTICLES YOU MAY BE INTERESTED IN}

Polarization-independent phase modulation using a polymer-dispersed liquid crystal Applied Physics Letters 86, 141110 (2005); https://doi.org/10.1063/1.1899749

Polarization-independent phase modulation of a homeotropic liquid crystal gel Applied Physics Letters 87, 191106 (2005); https://doi.org/10.1063/1.2126107

Polarizer-free and fast response microlens arrays using polymer-stabilized blue phase liquid crystals

Applied Physics Letters 96, 113505 (2010); https://doi.org/10.1063/1.3360860

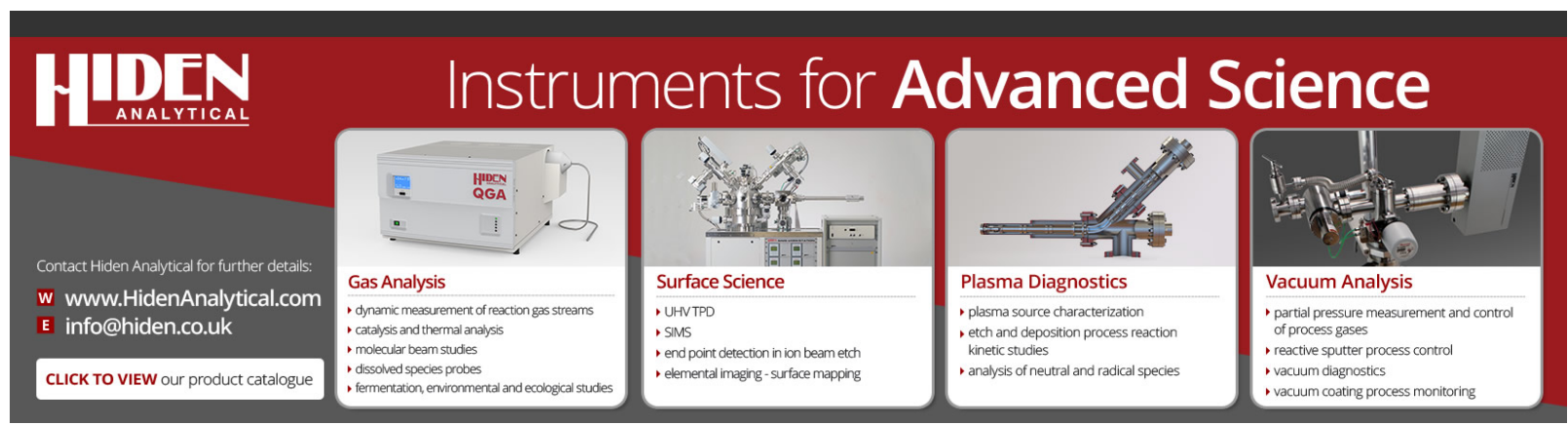




\title{
Polarization-independent and fast-response phase modulation using a normal-mode polymer-stabilized cholesteric texture
}

\author{
Yi-Hsin Lin, Hongwen Ren, Yun-Hsing Fan, Yung-Hsun Wu, and Shin-Tson Wu ${ }^{\text {a) }}$ \\ College of Optics and Photonics, University of Central Florida, Orlando, Florida 32816
}

(Received 9 May 2005; accepted 25 July 2005; published online 25 August 2005)

Fast-response, polarization-independent, and hysteresis-free phase-only modulation using a normal-mode polymer-stabilized cholesteric texture (PSCT) is demonstrated. Although the remaining phase change in the high-voltage regime is small, it is still useful for making microdevices. Polarization-independent tunable-focus microlens arrays using such a PSCT are demonstrated. (c) 2005 American Institute of Physics. [DOI: 10.1063/1.2037191]

\section{INTRODUCTION}

Polarization-independent and fast-response phase modulation is particularly desirable for photonic devices such as tunable-focus lens, grating, prism, and spatial light modulators. The most commonly employed phase-only liquidcrystal (LC) modulator is the homogeneous-aligned cell. The homogeneous cell provides a large phase change (normally $2 \pi$ ), however, this phase change is polarization sensitive and the response time which depends on the cell gap is relatively slow $(\sim 10 \mathrm{~ms})$. The use of a linear polarizer reduces the light transmission by more than $50 \%$.

Only a few approaches for achieving polarizationindependent phase modulation have been discovered, for instance, the $90^{\circ}$ twisted nematic cell operated at a voltage 3 $\times$ higher than the threshold voltage, ${ }^{1,2}$ nanoscale polymerdispersed liquid crystal (nano-PDLC), ${ }^{3}$ and PDLC at a voltage higher than the saturation voltage $\left(V_{s}\right){ }^{4}$ A common problem of these methods is that the phase change is relatively small and the operating voltage is quite high. Although the phase change is small, it is still useful for making microdevices such as tunable-focus microlens arrays. Thus, more polarization-independent light modulation mechanisms need to be developed.

In this paper, we find another polarization-independent phase modulation mechanism using a polymer-stabilized cholesteric texture (PSCT). PSCT has been studied for display and privacy window applications for more than a decade. ${ }^{5,6}$ Both normal-mode ${ }^{7}$ and reversed-mode ${ }^{8-12}$ PSCT devices have been developed. Usually a normal-mode PSCT is operated at a voltage below the saturation voltage where the maximum transmittance is first reached. In this paper, we find that a small phase modulation is available in the $V$ $>V_{s}$ region. Moreover, this phase change is polarization independent, hysteresis free, and has submillisecond response time. To demonstrate the usefulness of this polarizationindependent phase modulation, we fabricated microlens arrays based on the above-mentioned PSCT. The focal length is tunable from 3.5 to $5 \mathrm{~cm}$ when the voltage is switched from 180 to $80 V_{\text {rms }}$.

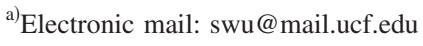

\section{EXPERIMENT}

To prepare a normal-mode PSCT cell, we mixed nematic LC E44 ( $\Delta n=0.26$, Merck), CB15 (a chiral agent), and a diacrylate monomer (bisphenol-A-dimethacrylate) at 90.25 : 5.75: $4 \mathrm{wt} \%$ ratios. The mixture was injected into an empty cell whose inner surfaces were coated with a thin indium-tinoxide (ITO) electrode only. The cell gap was controlled at $d=25 \mu \mathrm{m}$. During UV exposure, the filled cell was biased at $V=40 V_{\text {rms }}$ (1-kHz sinusoidal waves) so that the LC directors and monomer were reoriented nearly perpendicular to the substrates. The UV $(\lambda \sim 365 \mathrm{~nm})$ intensity was controlled at $I \sim 15 \mathrm{~mW} / \mathrm{cm}^{2}$ and the exposure time was $1 \mathrm{~h}$. The UV curing process took place at room temperature $\left(T \sim 23^{\circ} \mathrm{C}\right)$.

Figure 1 illustrates the operation mechanism of a

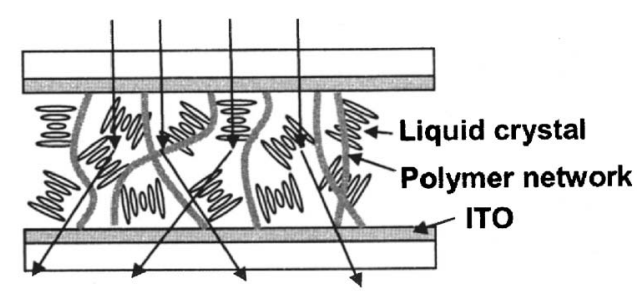

(a)

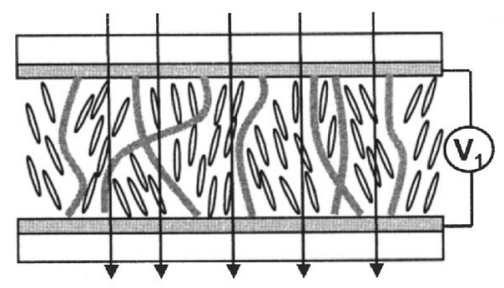

(b)

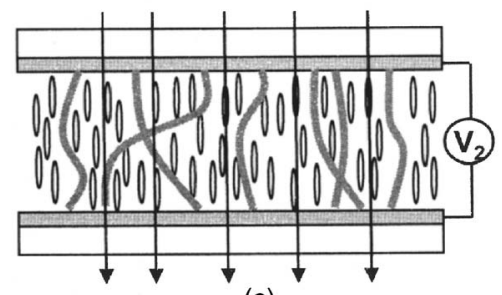

(c)

FIG. 1. The operation mechanism of PSCT at (a) $V=0$, (b) $V_{1} \sim V_{S}$, and (c) $V_{2}>V_{1}$. The residual phase between (b) and (c) can be used for phase modulator. 


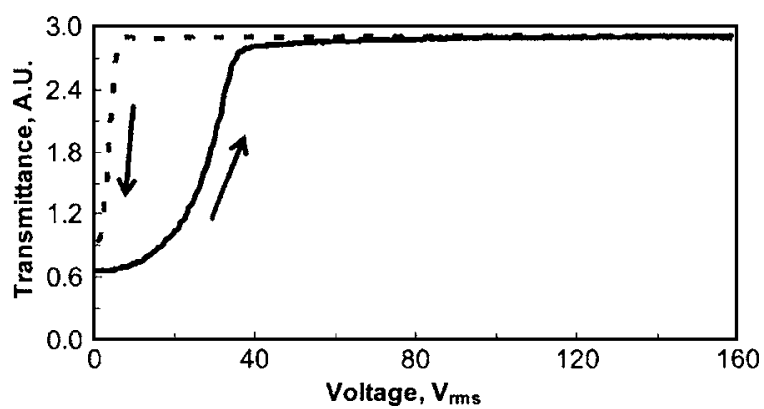

FIG. 2. The voltage-dependent transmittance of a PSCT cell with increasing voltage (black line) and decreasing voltage (dotted line).

normal-mode PSCT. To achieve a normal-mode operation, the LC cell was illuminated by an UV light in the homeotropic state with the presence of a bias voltage $\left(40 V_{\mathrm{rms}}\right)$. When the polymerization process is completed, the applied voltage is removed and then the focal conic texture is formed owning to the competition between the intrinsic spiral structure and the polymer constraint, as shown in Fig. 1(a). In this state, the cell is translucent because of strong light scattering originated from the polydomain focal conic structures. As the voltage reaches the saturation voltage, the electric field unwinds and transforms the spiral LC structures into a nearly homeotropic state. Under such a circumstance, the PSCT cell is transparent, as shown in Fig. 1(b). As the voltage exceeds $V_{S}$, more LC directors are aligned vertically, as shown in Fig. 1(c). Although the phase change between Figs. 1(b) and 1(c) is small, it is scattering free, polarization independent, hysteresis free, and has a fast response time.

\section{RESULTS AND DISCUSSION}

In experiment, we measured the transmittance of the PSCT cell using an unpolarized He-Ne laser $(\lambda=633 \mathrm{~nm})$ beam. No polarizer was used. A large-area photodiode detector was placed at $\sim 30 \mathrm{~cm}$ behind the sample, which corresponds to an $\sim 2^{\circ}$ collection angle. A computer-controlled LABVIEW data acquisition system was used for driving the sample and recording the light transmittance. Figure 2 plots the voltage-dependent transmittance of the normal-mode PSCT. The PSCT shows a severe hysteresis effect when the voltage is ramped up (black line) and down (dotted line). In the low-voltage regime, the PSCT scatters light strongly so that the transmittance is low and the sample appears translucent. As the voltage reaches the saturation voltage $V_{S}$ $\sim 40 V_{\text {rms }}$, the PSCT cell becomes highly transparent. In general, the saturation voltage is determined by the chiral dopant and monomer concentration, LC material, and cell gap. As shown in Fig. 2, the hysteresis is negligible when $V \geqslant V_{S}$.

\section{A. Phase change}

To characterize the phase change of PSCT, we measured the transmittance of the PSCT cell under crossed- and parallel-polarizer conditions. Results are plotted in Fig. 3. Below $40 V_{\text {rms }}$, the cell scatters light. Beyond $40 V_{\text {rms }}$, the transmittance $\left(T_{\perp}\right)$ for the crossed polarizers gradually decreases and $T_{\|}$increases for the parallel polarizers. This is a clear evidence that the phase modulation exists when the

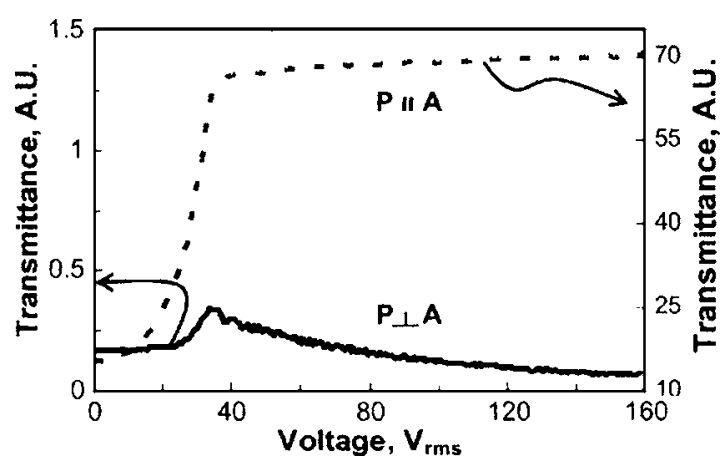

FIG. 3. The voltage-dependent transmittance of a PSCT cell between crossed polarizers (black line, left ordinate) and parallel polarizers (dotted line, right ordinate). $f=1 \mathrm{kHz}, \lambda=633 \mathrm{~nm}$, and cell gap $d=25 \mu \mathrm{m}$.

applied voltage exceeds the saturation voltage. Besides, when we rotate the PSCT cell between the polarizers, the transmittance curves remain unchanged. That means the PSCT phase modulator is indeed polarization independent.

The phase change $(\delta)$ of the PSCT cell can be calculated from following equation: ${ }^{13}$

$$
\delta=2 \tan ^{-1} \sqrt{T_{\perp} / T_{\|}},
$$

where $T_{\perp}$ and $T_{\|}$represent the transmittance under the crossed- and parallel-polarizer configurations, respectively. Figure 4 depicts the measured voltage-dependent phase change of the PSCT cell. The available phase decreases gradually with increased voltage because the LC directors are continuously reoriented by the electric field, as schematically shown in Fig. 1(c). The total phase change between 40 and $160 V_{\mathrm{rms}}$ is $\sim 0.025 \pi$. A higher voltage is required for getting more phase change. Although the residual phase change is small, it is still useful for making microdevices such as microlens arrays, micrograting, and microprism.

The residual phase can be increased by using a higher birefringence LC, larger cell gap, higher LC concentration, or higher curing temperature. The first two factors are obvious, but the last two needs some explanations. Based on our experimental results, the phase can be boosted by $\sim 2 \times$ as we increase the LC concentration from 90.25 to $93.61 \mathrm{wt} \%$ while reducing the chiral dopant from 5.75 to 3.39 wt $\%$ and polymer from 4 to $3 \mathrm{wt} \%$. The reduction of chiral dopant and polymer lowers the saturation voltage. As a result, the remaining phase increases. The tradeoff is the increased hysteresis. The curing temperature also has an important effect on the residual phase. At a given curing voltage, the domain

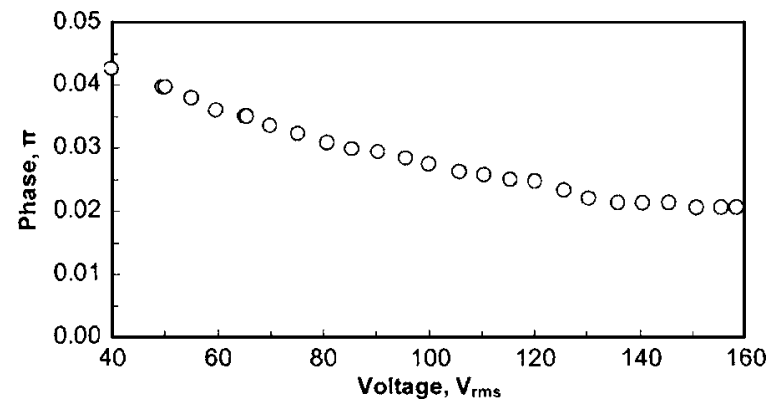

FIG. 4. Measured voltage-dependent phase shift of the E44 PSCT cell we prepared. Cell gap $d=25 \mu \mathrm{m}$. 

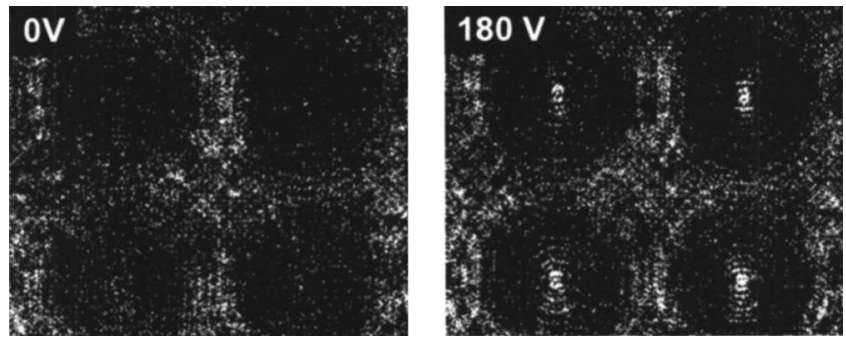

FIG. 5. Measured CCD images of $2 \mathrm{D}$ microlens arrays at $V=0$ and $V$ $=180 V_{\text {rms }}$.

size increases as the curing temperature increases ${ }^{14}$ which, in turn, lowers the saturation voltage. Therefore, the remaining phase increases. Our experimental results show that the phase is increased by $\sim 2-3 \times$ when the curing temperature is increased by $\sim 20^{\circ} \mathrm{C}$.

\section{B. Response time}

Next, we measured the response time of our PSCT phase modulator by using a square-wave voltage burst at $f$ $=10 \mathrm{kHz}$ between 40 and $160 V_{\text {rms }}$. The measured rise time is $\sim 75 \mu$ s and decay time is $\sim 793 \mu$ s. The very fast rise time originates from the high operating voltage $\left(160 V_{\text {rms }}\right)$ and the submillisecond decay time from the relatively high bias voltage $\left(40 V_{\text {rms }}\right)$ and the twist power of the chiral dopant and the polymer network.

\section{Microlens arrays}

To demonstrate the usefulness of this small residual phase change, we made a two-dimensional (2D) microlens array using the normal-mode PSCT as electro-optics medium. The fabrication method is similar to that reported earlier. ${ }^{15}$ First, we used the lamination method to prepare a polymer microlens arrays with NOA65 (Norland, $n_{p}=1.524$ ). The radius of curvature and the radius of aperture of each microlens are $\sim 950$ and $222 \mu \mathrm{m}$, respectively. Second, we filled our LC/polymer/chiral mixture into the polymer microlens array cavities. Third, the cell was sealed with a top ITO-glass substrate. Finally, the cell was biased at $\sim 140 V_{\text {rms }}$ during the UV curing process.

To characterize the focusing properties, a collimated unpolarized $\mathrm{He}-\mathrm{Ne}$ laser beam was used to illuminate the PSCT microlens arrays and then a charge-coupled device (CCD) camera was used for detecting the transmitted light. The focusing properties of the PSCT microlens arrays at 0 and $180 V_{\text {rms }}$ are shown in Fig. 5. At $V=0$, the imaging property is obscured because of the strong light scattering. At $V$ $=180 V_{\mathrm{rms}}$, clear focal spots appear. Moreover, the focal length of the patterned microlens arrays is tunable by the applied voltage. The focal length is $3.5 \mathrm{~cm}$ at $V=180 V_{\text {rms }}$ and increases to $5 \mathrm{~cm}$ at $V \sim 80 V_{\text {rms }}$. Based on this result, we can calculate the effective refractive index of the PSCT using the following equation:

$$
f=R^{2} / 2 d\left(n_{\text {eff }}-n_{p}\right),
$$

where $f$ is focal length of the lens, $R$ is the radius of the microlens aperture, $d$ is the LC thickness (the maximum

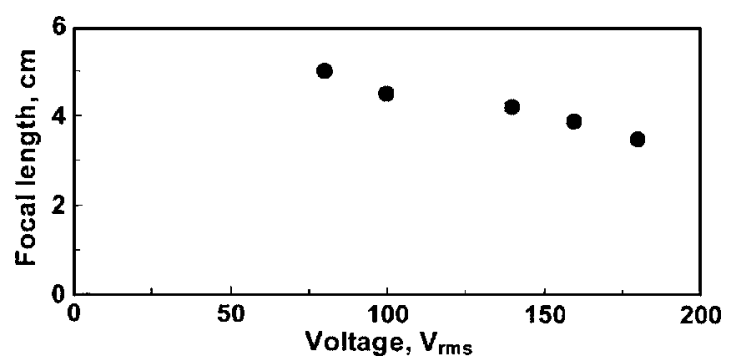

FIG. 6. Voltage-dependent focal length of the PSCT-based 2D microlens arrays.

thickness is $\sim 26 \mu \mathrm{m}$ ), and $n_{\text {eff }}$ is the effective refractive index of the PSCT. Plugging in the known values of $f, R, d$ and $n_{p}$ to Eq. (2), we find $n_{\text {eff }} \sim 1.551$ at $180 V_{\text {rms }}$. This value is reasonable because it should satisfy the following relationship: $n_{0}<n_{\text {eff }}\left(V_{\text {rms }}\right)<\left(2 n_{0}+n_{e}\right) / 3$, where $n_{0}=1.53$ and $n_{e}$ $=1.79$. From the above equation, the focal length of a microlens depends on the lens radius and thickness and the LC effective birefringence. To obtain a shorter focal length, we could reduce the lens diameter, use a higher birefringence $\mathrm{LC}$, or increase the LC lens thickness. The shortcoming of using a thick LC layer is the increased operating voltage. A simpler solution is to employ a high birefringence and lowthreshold-voltage PSCT.

Figure 6 plots the voltage-dependent focal length of the PSCT microlens arrays. The focal length of our lens array is polarization independent and is tunable from 3.5 to $5 \mathrm{~cm}$ when the voltage is switched from 180 to $80 V_{\text {rms. }}$.

The PSCT-based phase modulator exhibits an $\sim 4 \times$ lower threshold voltage $(\sim 1.6 \mathrm{~V} / \mu \mathrm{m})$ than the nano-PDLC phase modulator $^{3}(\sim 6 \mathrm{~V} / \mu \mathrm{m})$ because of its larger domain size. The response time is somewhat slower but still comparable to that of nano-PDLC phase modulators. In comparison to a conventional PDLC phase modulator, ${ }^{4}$ the PSCT exhibits a faster response time because the chiral dopant provides an extra force to assist the LC relaxations. However, it is also due to the chiral dopant the PSCT threshold voltage is higher than that of a PDLC phase modulator.

\section{CONCLUSION}

We have demonstrated a polarization-independent, scattering-free, hysteresis-free, and fast-response PSCTbased phase modulator. Although the operating voltage is high and the residual phase is small, the PSCT is still useful for making microdevices. PSCT-based tunable-focus microlens arrays are demonstrated.

\section{ACKNOWLEDGMENTS}

The authors would like to thank Chi Mei Optoelectronics (Taiwan) for providing the ITO glass substrates. This work is supported by DARPA BOSS program under Contract No. DAAD 19-02-1-0208.

${ }^{1}$ J. S. Patel, Appl. Phys. Lett. 58, 1314 (1991).

${ }^{2}$ Y. Huang, T. X. Wu, and S. T. Wu, J. Appl. Phys. 93, 2490 (2003).

${ }^{3}$ R. L. Sutherland, V. P. Tondiglia, L. V. Natarajan, T. J. Bunning, and W. W. Adams, Appl. Phys. Lett. 64, 1074 (1994).

${ }^{4}$ H. Ren, Y. H. Lin, Y. H. Fan, and S. T. Wu, Appl. Phys. Lett. 86, 141110 
(2005).

${ }^{5}$ D. K. Yang, L. C. Chien and Y. K. Fung, Liquid Crystals in Complex Geometries Formed by Polymer and Porous Networks (Taylor \& Francis, London, 1996).

${ }^{6}$ S. T. Wu and D. K. Yang, Reflective Liquid Crystal Displays (Wiley, New York, 2001).

${ }^{7}$ D. K. Yang, L. C. Chien, and J. W. Doane, Appl. Phys. Lett. 60, 3102 (1992).

${ }^{8}$ J. L. West, R. B. Akins, J. Francl, and J. W. Doane, Appl. Phys. Lett. 63 1471 (1993).
${ }^{9}$ I. Dierking, L. L. Kosbar, A. Afzali-Ardakani, A. C. Lowe, and G. A. Held, J. Appl. Phys. 81, 3007 (1997).

${ }^{10}$ I. Dierking, L. L. Kosbar, A. C. Lowe, and G. A. Held, Liq. Cryst. 24, 387 (1998).

${ }^{11}$ I. Dierking, L. L. Kosbar, A. C. Lowe, and G. A. Held, Liq. Cryst. 24, 397 (1998).

${ }^{12}$ H. Ren and S. T. Wu, J. Appl. Phys. 92, 797 (2002).

${ }^{13}$ S. T. Wu, U. Efron, and L. D. Hess, Appl. Opt. 23, 3911 (1984).

${ }^{14}$ F. Du and S. T. Wu, Appl. Phys. Lett. 83, 1310 (2003).

${ }^{15}$ H. Ren, Y. H. Fan, and S. T. Wu, Opt. Lett. 29, 1608 (2004). 